Results 629 patients qualified for the study. Median age was 64 [54-75], median NIHSS was 16 [12.5-21] and median time from last-known normal to CT was 317 mins [193-578]. $161(25.6 \%)$ presented with a TMC. On univariate analysis a TMC was associated with isolated deep MCA strokes $(77.6 \%$ vs $56.6 \%, \mathrm{p}<0.001)$ and higher collateral scores $(3[2-3]$ vs 2 [1-3], $\mathrm{p}<0.001)$. There were no differences between patients presenting with a TMC and others in good outcomes (mRS $0-2)(50.8 \%$ vs $47.6 \%, \mathrm{p}=0.53)$ or 90 -day mortality $(23 \%$ vs $17.6 \%, \mathrm{p}=0.17$ ) while TMC patients had lower rates of any parenchymal hematomas $(5.2 \%$ vs $14.6 \%, p=0.02)$. After adjusting for potential confounders, isolated deep MCA strokes was identified as an independent predictor of TMC (OR 2.49 95\%CI 1.63-3.8, p<0.001).

Conclusions Our results show that CT perfusion might miss infarct core in $25 \%$ of the case, especially in isolated deep MCA strokes. In those cases, a CT Perfusion based paradigm to determine eligibility for ET might not be as accurate. Future studies looking into the optimal selection tools for this patient population as well as refinements of the post-processing algorithms addressing this pitfall are warranted.

Disclosures M. Bouslama: None. K. Ravindran: None. G. Rodrigues: None. L. Pisani: None. D. Haussen: None. C. Barreira: None. M. Frankel: None. R. Nogueira: 2; C; Stryker Neurovascular (Trevo-2 Trial PI - modest, DAWN Trial PI - no payment, Trevo Registry Steering Committee - no payment, Physician Advisory Board/Consultant - significant), Covidien/Medtronic (SWIFT and SWIFT-PRIME Steering Committee - modest, STAR Trial Core Lab - significant), Neuravi/ Cerenovus (ARISE-II Trial Steering Committee - no payment, Physician Advisory Board - modest), Phenox (Physician Advisory Board/Consultant - modest), Genentech (Physician Advisory Board/Consultant - modest). 6; C; Penumbra (3-D Separator Trial Executive Committee - no payment).

\section{E-058 LATEST ADVANCEMENTS IN CONE BEAM CT IMPROVE VISUALIZATION OF EARLY ISCHEMIC BRAIN PARENCHYMAL CHANGES DURING STROKE}

${ }^{1} \mathrm{~N}$ Cancelliere*, ${ }^{1} \mathrm{P}$ Nicholson, ${ }^{1} \mathrm{~J}$ Bracken, ${ }^{2} \mathrm{~F}$ Nijnatten, ${ }^{2} \mathrm{E}$ Hummel, ${ }^{2} \mathrm{~T}$ Grunhagen, ${ }^{2} \mathrm{M}$ Vlimmeren, ${ }^{1} \mathrm{~T}$ Krings, ${ }^{1} \mathrm{~V}$ Mendes Pereira. ${ }^{1} J$ oint Department of Medical Imaging, University Health Network, Toronto, ON, Canada; ${ }^{2}$ Philips Healthcare, Best, Netherlands

\subsection{6/neurintsurg-2019-SNIS.133}

Background and purpose Time is essential for treatment of acute ischemic stroke (AIS) for patients with large-vessel occlusion (LVO). It is now well established that decreasing the time between stroke-onset to groin-puncture significantly improves functional outcomes for these patients (Bourcier et al., 2019). One potential method of shortening this time is to by-pass the CT department and bring patients directly to the angiosuite for baseline imaging. However, in order for this to gain mainstream acceptance, the diagnostic quality of cone beam CT performed in the angiosuite needs to be comparable to that of conventional CT to exclude haemorrhages and well-define the stroke core. Therefore, the purpose of this study was to compare the imaging quality of the latest generation cone beam CT (CB-CT) imaging to conventional multidetector CT (MD-CT) using a quantitative and qualitative analysis

Methods Patients with AIS who received endovascular mechanical thrombectomy were prospectively included in this monocentric study $(\mathrm{n}=55)$. Baseline MD-CT imaging acquired in CT was compared to one of two CB-CT imaging protocols acquired in the angiosuite: either the traditional CB-CT imaging protocol or the newest generation CB-CT imaging protocol, which has a faster acquisition time and improved built-in reconstruction algorithms. All three imaging datasets were analyzed using quantitative and qualitative measures. Average houndsfield units and standard deviations were calculated in 4 gray and 4 white matter regions and a contrast-to-noise ratio (CNR) was calculated. Two neuroradiologists with varying levels of expertise assessed 6 measures of image quality (Noise, Artefacts, Gray/white matter differentiation, Subarachnoid space sharpness, Ventricular margins, Distinctiveness of posterior fossa contents) using a 5-point Likert-scale $(1=$ Very poor, $2=$ Poor, $3=$ Acceptable, $4=$ Good; $5=$ Very Good). Diagnosis of early ischemic changes were quantified using ASPECTS and scores were compared between groups.

Results Mean CNR in MD-CT $(2.86 \pm 0.72)$ was superior to CB-CT imaging $(2.24 \pm 0.87 ; \mathrm{p}=<0.01)$; however, after dose correction (MD-CT $=105 \mathrm{mGy}$ vs. CB-CT $=65 \mathrm{mGy}$ ), the difference in mean CNR values between both groups was eliminated (CB-CT dose corrected $\mathrm{CNR}=2.93 \pm 1.29$; $\mathrm{p}=0.58)$. Kruskal-Wallis one-way analysis of variance with follow-up Dunn's tests revealed that although MD-CT is superior to CB-CT image quality, the latest generation CBCT imaging protocol provides images that are consistently superior to the traditional CB-CT imaging protocol in all 6 categories scored. New CB-CT images had less noise $(\mathrm{p}=$ $<0.001)$ and artefacts $(p=<0.01)$, and better gray/white matter differentiation $(\mathrm{p}=<0.001)$, subarachnoid space sharpness $(p=<0.001)$, ventricular margins $(p=<0.01)$ and distinctiveness of Posterior fossa contents $(p=<0.02)$ compared to traditional CB-CT. Additionally, 90.3\% of new CBCT $(n=28 / 31)$ scans were deemed acceptable to give an ASPECTS score, compared to only $50 \%$ of traditional CBCT $(n=3 / 6)$ images.

Conclusions The latest generation of CB-CT images are superior to older CB-CT imaging protocols, having superior quantitative and qualitative features. Although MD-CT is still superior, recent improvements suggest that CB-CT is acceptable for emergency stroke imaging assessment before mechanical thrombectomy, which reduces door-to-groin puncture times and improve patient outcomes.

Disclosures N. Cancelliere: None. P. Nicholson: None. J. Bracken: 5; C; Philips Healthcare. F. Nijnatten: 5; C; Philips Healthcare. E. Hummel: 5; C; Philips Healthcare. T. Grunhagen: 5; C; Philips Healthcare. M. Vlimmeren: 5; C; Philips Healthcare. T. Krings: None. V. Mendes Pereira: 1; C; Philips Healthcare. 2; C; Stryker, Medtronic.

\section{E-059 DURAL VENOUS SINUS CEPHALOCELES IN THE PULSATILE TINNITUS CLINIC}

1J Villanueva-Meyer*, ${ }^{2} \mathrm{~L}$ Eisenmenger, ${ }^{1} \mathrm{~V}$ Shah, ${ }^{3} \mathrm{~K}$ Meisel, ${ }^{1} \mathrm{M}$ Amans. ${ }^{1}$ Radiology and Biomedical Imaging, UCSF, San Francisco, CA; ${ }^{2}$ Radiology, University of Wisconsin, Madison, WI; ${ }^{3}$ Neurology, UCSF, San Francisco, CA

\subsection{6/neurintsurg-2019-SNIS.134}

Brain herniations into the dural venous sinuses are recently described rare findings that are of uncertain etiology and clinical significance. We describe 18 instances of brain herniations 